\title{
Note sur quelques Acariens trouvés sur Sorex araneus (Mamm., Insectivora).
}

\author{
par G. L. van EYNDHOVEN
}

La présente note a pour objet de signaler la présence de «corpora aliena », enkystées dans la peau de petits Mammifères, phénomène peu fréquent, d'après les informations reçues.

Une musaraigne carrelet, Sorex araneus araneus L., capturée le 25 avril 1957 dans les environs d'Amsterdam, me fut remise par M. M. Servaas. Sur le dos, l'animal présentait une petite plaque d'aspect chauve, mesurant environ $6 \times 5 \mathrm{~mm}$. Les poils longs du pelage avaient disparu, et il ne restait qu'un grand nombre de poils très courts. Au centre de la plaque apparaissait un petit bourgeon de $2 \times 1,5 \mathrm{~mm}$ couvert d'une escarre. En soulevant cette escarre, je vis un kyste séparé du tissu normal par une paroi épaisse. Dans la cavité kystique, d'un diamètre de $0,75 \mathrm{~mm}$, se trouvaient deux Acariens chitinisés, morts mais bien conservés et entiers, appartenant aux Oribatei et tous deux à l'espèce Punctoribates punctum (C. L. Koch, 1839). Pour le reste, la cavité était vide.

Les Oribatei, étant normalement des Acariens phytophages, n'ont à priori aucune raison de parasiter l'animal. Ils vivent habituellement dans les mousses, sur les lichens, etc., et beaucoup d'espèces sont rencontrées dans l'épaisseur du sol. Pénétrer dans les nids et les galeries de petits Mammifères est chose facile pour de tels animalcules. Parmi de nombreuses espèces d'Acariens observées dans les nids de Microtus guentheri Danford et Alston (Levant Vole en anglais), Costa (1961) a trouvé, en Israël, trois exemplaires de ce même Punctoribates punctum.

Pour interpréter le développement du kyste observé chez Sorex araneus, on peut supposer qu'une lésion préalable existait et que, par une coïncidence, les deux Punctoribates ont été capturés dans cette blessure ouverte, et ultérieurement enkystés comme des corps étrangers.

Etant donné la possibilité de la présence d'autres organismes parasitaires, comme le Demodex ou les Acariens de la gale (Sarcoptes, Notoedres), j'ai examiné la peau autour du bourgeon, mais sans résultat. Sachant par ailleurs, que le nez des petits Mammifères est l'habitat favori des Demodex, Acariens des follicules, et afin de disposer d'un élément de comparaison, j'ai examiné aussi cet organe. Quelques exemplaires de Demodex soricinus Hirst, 1918 s'y trouvaient, dont la présence et la localisation peuvent être considérées comme normales ; ils n’y déterminent pas de lésions. 
Demodex soricinus a pour hôte spécifique Sorex araneus. Hirst (1918: 45, 1919 : 37, pl. 11, fig. 48) l'a décrit chez la sous-espèce Sorex araneus castaneus Jenyns, en Grande-Bretagne. Les Acarologues n'ayant pas souvent étudié la famille des Demodicidae, il ne semble pas que Demodex soricinus ait été déjà signalé chez la sous-espèce continentale Sorex araneus araneus L.

Dans leur pelage, les musaraignes hébergent une petite faune d'autres espèces d'Acariens qui, normalement, sont parfaitement tolérées. Parmi celles-ci, les membres de la sous-famille des Myobiinae, appartenant aux Cheyletidae, sont des épizoa réguliers. Aux Pays-Bas, on trouve deux espèces sur la musaraigne carrelet: Protomyobia claparedei (Poppe, 1896) et Amorphacarus elongatus (Poppe, 1896). On les voit souvent ensemble, hébergés par le même animal, et leur fréquence et leur nombre sont à peu près identiques.

C'est Ewing (1938: 191) qui a créé le nouveau genre Amorphacarus pour Myobia elongata Poppe, en se basant e.a. sur l'asymétrie de la partie antérieure du corps et de la première paire de pattes. Une de ces pattes est réduite et ce caractère se manifeste chez les adultes, les nymphes et probablement aussi chez les larves. Radford (1935: 252,1949 : 93) signale que c'est toujours la première patte de droite qui est réduite. Dans le matériel que j'ai à ma disposition, cette réduction se produit aussi bien à gauche qu'à droite, et les deux formes sont généralement présentes dans la même population. Je dispose de 51 Acariens, récoltés au hasard sur 8 musaraignes de localités diverses. Voici la répartition des réductions observées :

\begin{tabular}{|c|c|c|c|c|c|c|c|}
\hline \multicolumn{8}{|c|}{ Réduction } \\
\hline$\underset{\mathrm{N}^{\circ}}{\text { Hôte }}$ & & \multicolumn{2}{|c|}{$\begin{array}{c}\text { MaLe } \\
\text { gauche droite }\end{array}$} & \multicolumn{2}{|c|}{$\begin{array}{c}\text { Femelle } \\
\text { gauche droite }\end{array}$} & \multicolumn{2}{|c|}{$\begin{array}{l}\text { NympHe } \\
\text { gauche droite }\end{array}$} \\
\hline 1 & $\ldots \ldots \ldots \ldots \ldots \ldots \ldots \ldots \ldots \ldots \ldots$ & 1 & 2 & 1 & 2 & 1 & - \\
\hline 2 . & 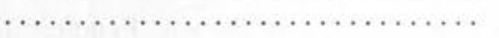 & - & - & - & - & 一 & 3 \\
\hline 3. & n.m. & - & 1 & - & - & - & $\overline{1}$ \\
\hline 4. & 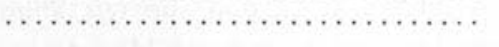 & 1 & - & - & - & - & 1 \\
\hline 5 & 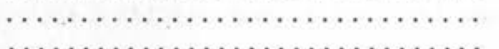 & 2 & - & 1 & 1 & 一 & 一 \\
\hline 6 & 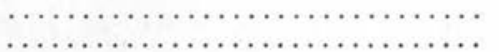 & $\begin{array}{l}4 \\
8\end{array}$ & $\begin{array}{l}1 \\
2\end{array}$ & $\begin{array}{l}3 \\
3\end{array}$ & $\frac{2}{-}$ & $\overline{7}$ & $\overline{1}$ \\
\hline $\begin{array}{l}7 \\
8\end{array}$ & 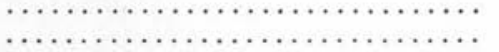 & 1 & 1 & $\begin{array}{l}3 \\
1\end{array}$ & 二 & - & 1 \\
\hline
\end{tabular}

Dans ce matériel limité, 34 exemplaires présentent donc une réduction à gauche et 17 à droite.

Aux Etats-Unis, Sorex cinereus Kerr (Masked Shrew) et Sorex fumeus Miller (Smoky Shrew) hébergent un Amorphacarus voisin, l'Amorphacarus hengererorum Jameson, 1948. Ewing (1938) fut le premier à signaler cette espèce chez Sorex cinereus et, la considérant comme identique à celle d'Europe, selon la description de Poppe, il la décrivit sous le même nom spécifique elongatus. Jameson (1948) découvrit des différences dans les caractères morphologiques et en fit une nouvelle espèce : Amorphacarus hengererorum. Selon ces deux auteurs, l'espèce américaine a une préférence marquée pour la réduction de la première patte de gauche. Ewing $(1938$ : 192) avait à sa disposition cinq exemplaires provenant de Sorex cinereus de deux localités, dont quatre 
montraient une réduction de gauche. Jameson (1948: 340) y ajouta 25 exemplaires récoltés dans deux localités sur Sorex fumeus et trois exemplaires provenant d'une seule localité prélevés sur Sorex cinereus ; ces exemplaires montraient également presque tous une réduction à gauche.

\section{Résumé}

Les Acariens suivants, tous récoltés sur Sorex araneus, sont étudiés :

1. Deux Punctoribates punctum enkystés dans la peau dorsale ;

2. Demodex soricinus prélevé dans la peau du nez;

3. Protomyobia claparedei et Amorphacarus elongatus découverts dans le pelage ;

4. L'asymétrie de la première paire de pattes est signalée chez Amorphacarus elongatus et $A$. hengererorum.

\section{Bibliographie}

Costa (Michael), 1961. - Mites recovered from the nests of the Levant Vole (Microtus guentheri) in Israel. Ann. Mag. nat. Hist., sér. 13, vol. 4, $\mathrm{n}^{\circ} 41$, p. 257-282, fig. 1-75.

EwING (H. E.), 1938. - North American mites of the subfamily Myobiinae, new subfamily (Arachnida). Proc. ent. Soc. Washington, vol. 40, $\mathrm{n}^{\circ} 7,27-10-1938$, p. 180-197, fig. 1.

HrRSt (Stanley), 1918. - On Four new Species of the Genus Demodex, Owen. Ann. Mag. nat. Hist., sér. 9, vol. 2, $\mathrm{n}^{\circ}$ 8, 8-1918, p. 145-146.

-, 1919. - The Genus Demodex, Owen. Brit. Mus. (nat. Hist.), Studies on Acari, $\mathrm{n}^{\circ} 1$, p. I-III, 1-44, 4 fig. in texte, pl. I-XIII, fig. 1-53.

JAMESON Jr. (E. W.), 1948. - Myobiid mites (Acarina : Myobiinae) from Shrews (Mammalia: Soricidae) of Eastern North America. Journ. Parasit., vol. 34, $\mathrm{n}^{\circ} 4,8-1948$, p. 336342 , fig. 1-2.

Косн (C. L.), 1839. - Deutschlands Crustaceen, Myriapoden und Arachniden. Regensburg, fasc. $30, \mathrm{n}^{\circ} 22,1-11-1839$.

POPPE (S. A.), 1896. - Beitrag zur Kenntnis der Gattung Myobia v. Heyden (Schluss). Zool. Anzeiger, vol. $19, \mathrm{n}^{\circ} 509,10-8-1896$, p. 337-349, fig. 7-25.

RADFORD (C. D.), 1935. - Notes on Mites of the Genus Myobia. North Western Naturalist, 9-1935, p. 248-258, fig. 1-22.

-, 1949. - A revision of the fur mites Myobiidae (Acarina) (Suite). Bull. Mus. nation. Hist. nat., sér. 2 , vol. $21, \mathrm{n}^{\circ} 1$ (janvier 1949), publ. 5-5-1949, p. 91-97, fig. 19-30.

(Zoölogisch Museum, Amsterdam) 